\title{
Cancro de Mama: Valor em Saúde, Custos e Financiamento
}

\section{Breast Cancer: Value-Based Healthcare, Costs and Financing}

\author{
Ana HARFOUCHE $\triangle^{1,2}$, Sílvia SILVA ${ }^{3,4}$, João FARIA ${ }^{5}$, Rui ARAÚJO ${ }^{6}$, António GOUVEIA ${ }^{7}$, Maria LACERDA ${ }^{8}$, \\ Luís D'OREY 5 \\ Acta Med Port 2017 Nov;30(11):762-768 - https://doi.org/10.20344/amp.9093
}

\section{RESUMO}

Introdução: O cancro de mama é a segunda doença oncológica mais comum no mundo. Com o propósito de estudar o novo financiamento por patologia - cancro de mama - implementado no Instituto Português de Oncologia de Lisboa Francisco Gentil, foram analisados os custos reais dos doentes, através da metodologia de custeio activity based costing. Pretendeu-se dar resposta à pergunta de investigação: "Quanto custa tratar o cancro de mama por 'mês*doente' face ao 'envelope financeiro' fixo mensal?".

Material e Métodos: $O$ universo foi constituído por 807 doentes correspondendo a todos os doentes elegíveis no novo programa de financiamento por patologia e entrados ao longo do primeiro ano de implementação. Através do activity based costing foram apurados os custos reais totais por estádio da doença e por 'mês*doente' e o desvio relativamente ao 'envelope financeiro' fixo mensal.

Resultados: Total de custos (6,6 M€), total de financiamento (5,2 M€) para um total de 5648 'meses*doente'. Em 2014, o saldo entre o financiamento obtido e os custos reais, foi negativo em 1,4 M€ para o universo de 807 doentes.

Discussão: As situações extremas em termos de custos por 'mês*doente' face ao 'envelope financeiro' mensal fixo foram: (i) o estádio O/TIS com financiamento superior em 415,23 € por 'mês*doente'; (ii) o estádio IIIC com um financiamento inferior em $1062,79 €$ por 'mês*doente'.

Conclusão: O custo 'mês*doente', independentemente do estádio da doença, foi de $1170,29 €$. O desvio médio 'mês*doente' foi negativo $(241,21 €)$ face ao 'envelope financeiro' mensal fixo de 929,08 € no primeiro ano. Estabelecer modelos de financiamento com base no activity based costing será crucial para a sustentabilidade futura do sector da saúde.

Palavras-chave: Análise Custo-Benefício; Custos e Análise de Custo; Custos em Saúde; Neoplasias da Mama/economia

\section{ABSTRACT}

Introduction: Breast cancer is the second most common oncological disease worldwide. To analyse the new disease specific funding programme (breast cancer) implemented at the Francisco Gentil Portuguese Institute of Oncology, Lisbon Center (Instituto Português de Oncologia de Lisboa Francisco Gentil), the actual costs of the patients were examined using activity-based costing as a costing methodology. This study addresses the following question: "How much does it cost to treat breast cancer per 'patient-month' compared to the monthly fixed 'funding envelope'?".

Materials and Methods: The study cohort consisted of 807 patients, corresponding to all the patients eligible for the new disease specific funding programme and who were enrolled during the first year of implementation. Activity-based costing was used to calculate the total real costs per stage of disease and per 'patient-month' as well as the deviation from the monthly fixed 'funding envelope'.

Results: The total costs were 6.6 M€, whereas the total funding was $5.2 \mathrm{M} €$ for a total of 5648 'patient-months'. In 2014 , the balance difference between the funding obtained and the actual costs was $-1.4 € \mathrm{M}$ for the cohort of 807 patients.

Discussion: The extreme cases of differences in cost per 'patient-month' compared to the monthly fixed 'funding envelope' were (i) stage $0 /$ Tis, with higher funding at $415.23 €$ per 'patient-month', and (ii) stage IIIC, with lower funding at $1062.79 €$ per 'patient-month'. Conclusion: The 'patient-month' cost, regardless of disease stage was $1170.29 €$. The median deviation per 'patient-month' was negative $(241.21 €)$ compared to the monthly fixed 'funding envelope' of $929.08 €$ in the first year. Establishing activity-based costing funding models will be crucial for the future sustainability of the healthcare sector.

Keywords: Breast Neoplasms/economics; Cost-Benefit Analysis; Costs and Cost Analysis; Health Care Costs

\section{INTRODUÇÃO}

Num contexto em que os custos associados ao sector da saúde continuam, cada vez mais, a representar uma parcela maior das receitas fiscais (em 2014 e segundo os dados do Ministério das Finanças, o orçamento do Serviço Nacional de Saúde (SNS) correspondeu a cerca de $63 \%$ das receitas em IRS, a $46 \%$ dos impostos diretos e a $22 \%$ do total de impostos), surge a necessidade de se criarem novas metodologias para o conhecimento dos custos reais envolvidos no diagnóstico e tratamento das diferentes patologias. Este conhecimento permitirá ajudar a fundamentar, no futuro, as opções políticas em saúde.

Em 2012, e segundo a Organização Mundial de Saúde, o cancro de mama foi responsável por 521000 mortes $^{1}$ no mundo, ou seja, aproximadamente $6,4 \%$ do total de mortes

1. Instituto Superior de Ciências Sociais e Políticas - Centro de Administração e Políticas Públicas (CAPP). Universidade de Lisboa. Lisboa. Portugal.

2. Concepção, Implementação e Desenvolvimento do Activity Based Costing por Actividades Clínicas. Instituto Português de Oncologia de Lisboa Francisco Gentil. Lisboa. Portugal.

3. Serviço de Cirurgia Geral. Hospital da Marinha Portuguesa. Lisboa. Portugal.

4. Serviço de Cirurgia Geral. Hospital Egas Moniz. Centro Hospitalar Lisboa Ocidental. Lisboa. Portugal.

5. Serviço de Cirurgia Geral. Instituto Português de Oncologia de Lisboa Francisco Gentil. Lisboa. Portugal.

6. Activity Based Costing por Actividades Clínicas. Instituto Português de Oncologia de Lisboa Francisco Gentil. Lisboa. Portugal.

7. Serviços Farmacêuticos. Instituto Português de Oncologia de Lisboa Francisco Gentil. Lisboa. Portugal.

8. Hospital de Dia. Instituto Português de Oncologia de Lisboa Francisco Gentil. Lisboa. Portugal.

$\square$ Autor correspondente: Ana Harfouche. aharfouche1@gmail.com

Recebido: 18 de abril de 2017 - Aceite: 12 de outubro de 2017 | Copyright @ Ordem dos Médicos 2017 
por cancro.

Em Portugal são ainda diagnosticados cerca de 6000 novos casos por ano, dos quais um em cada 100 é do sexo masculino. $^{2}$

De acordo com o Registo Oncológico Nacional, houve um aumento de $38 \%$ dos casos entre 2005 e 2007, no sexo feminino. Em conformidade com a American Cancer Society, os dados revelam também que uma em 35 mulheres diagnosticadas corre o risco de morrer, e que uma em cada oito tem a probabilidade de vir a ter cancro de mama ao longo da sua vida. ${ }^{3}$

Por ser este o cancro mundialmente mais frequente e também por ser o cancro com maior número de doentes elegíveis para o novo programa de financiamento por patologia, determinou-se que a pergunta de investigação seria a seguinte: "Quanto custa tratar o cancro de mama por 'mês*doente' face ao 'envelope financeiro' fixo mensal?".

O novo programa de financiamento por patologia, promovido pela Administração Central do Sistema de Saúde (ACSS) desde 2013, contempla três patologias específicas - (i) cancro de mama; (ii) cólon e recto e (iii) colo do útero.

No total de novos doentes incluídos nestas três patologias financiadas de forma específica no Instituto Português de Oncologia de Lisboa (IPO Lisboa), perfazendo 1228 doentes em 2014, 66\% dos mesmos apresentavam cancro de mama (807 doentes).

Este estudo de investigação tem como objetivo conhecer os custos reais 'mês*doente' na patologia cancro de mama, por ser aquela que apresentou maior número de doentes elegíveis de acordo com os critérios deste novo programa de financiamento, no primeiro ano de implementação do mesmo no IPO Lisboa, isto é, em 2014 e compará-los com o 'envelope financeiro' fixo mensal por doente.

O financiamento fixo atribuído por doente e por mês foi de 929,08 $€$, independentemente dos atos clínicos realizados aos doentes.

A unidade física utilizada para caracterizar o universo foi 'mês*doente'. Assim, foi possível comparar o financiamento obtido com os custos reais incorridos.

Utilizou-se a metodologia activity based costing $(A B C)^{4,5}$ correntemente designada por custeio $A B C$ e que tem vindo a ser desenvolvida no IPO Lisboa.

Surgiram dificuldades na pesquisa de artigos científicos idênticos ao presente estudo, uma vez que os artigos encontrados não são devidamente claros em relação ao período temporal da investigação. Também não foram encontrados estudos que desagreguem os custos por estádios tumor, node and metastasis (TNM). ${ }^{6}$ Ainda assim, foi identificado um estudo realizado no Reino Unido, ${ }^{7}$ ao longo de 15 meses (anos 2010 e 2011) com uma amostra de 223 pacientes com cancro da mama ao qual faremos referência nas conclusões, embora não permita comparações.

\section{MATERIAL E MÉTODOS}

O desenho utilizado para esta investigação assenta nas seguintes etapas:

$1^{\text {a) }}$ Estudo do universo dos doentes diagnosticados e que foram elegíveis de acordo com os critérios do novo programa de financiamento por patologia. Este novo programa apresenta quatro critérios para inclusão dos doentes, ou seja a elegibilidade dos doentes obedece a: $1^{\circ}$. Apenas são incluídos os doentes que iniciem tratamento na instituição em causa (são excluídos doentes parcialmente tratados em outras instituições, doentes em recidiva ou progressão); $2^{\circ}$. Apenas são incluídos doentes com tratamento dirigido ao cancro, ou seja, têm de ter uma das seguintes modalidades - cirurgia, quimioterapia, radioterapia ou hormonoterapia; $3^{\circ}$. São incluídos todos os tratamentos após a admissão do doente, independentemente da sua relação com a patologia, durante o período de dois anos (730 dias); $4^{\circ}$. São incluídos os procedimentos de diagnósticos, terapêuticos, complementares e de follow-up;

$2^{a}$ ) Horizonte temporal - O estudo engloba 807 doentes, todo o universo de doentes elegíveis para este financiamento específico por cancro de mama, os quais foram entrando ao longo do ano de 2014 (janeiro a dezembro). O financiamento e os custos foram calculados de acordo com os meses decorridos entre o mês em que o doente foi elegível e entrou para o novo financiamento por patologia e o último mês do ano 2014. Como exemplo: o doente que entrou em janeiro tem 12 meses de financiamento e de custos e o doente que entrou em maio tem 8 meses;

$3^{a}$ ) Estratificação dos doentes por estádios da doença;

$4^{a}$ ) Análise dos custos reais 'mês*doente' tendo em conta o consumo dos recursos ao longo da trajetória de diagnóstico e tratamento, nomeadamente consultas, meios complementares de diagnóstico e terapêutica (MCDT's), medicamentos, bloco operatório, sessões de hospital de dia e episódios de internamento;

$\left.5^{a}\right)$ Apuramento do desvio entre os custos reais 'mês*doente' e o financiamento fixo específico obtido para esta patologia.

O estudo dos custos reais do cancro de mama decorre do trabalho já desenvolvido no IPO Lisboa desde 2008 com o propósito de obter uma contabilidade de custos reais das atividades e dos consumos de recursos praticados em instituições de saúde. Esta metodologia foi promovida pela ACSS através de projetos-piloto em vários hospitais do país, dos quais se desconhecem os resultados destes ensaios piloto por não estarem publicados.

O projeto de custeio $A B C$ iniciado em 2008 permitiu, em 2012 e com a metodologia ABC, custear de forma automatizada todos os atos e atividades no IPO Lisboa. Através do custeio $A B C$ obtém-se, nomeadamente informação de quanto custa um dia de internamento em cada especialidade, uma sessão de hospital de dia, um medicamento, um exame de diagnóstico, uma sessão de fisioterapia, uma consulta de especialidade ou uma consulta multidisciplinar de decisão terapêutica.

A metodologia de custeio $A B C$ associada à base de dados Structured Query Language (SQL) possibilita obter, de forma automatizada, informação dos custos envolvidos por atos e por atividades realizadas, tendo em conta a estrutura de custos, as alocações de tempos por diferenciação 
profissional a cada ato e o respetivo consumo de recursos.

Esta metodologia apresenta um conhecimento que os sistemas de contabilidade analítica tradicionais não permitem por serem baseados em rateios de grandes agregados de custos. Por outro lado, tradicionalmente não especializam tempos e consumos a atos e atividades concretas, mas a secções homogéneas como por exemplo, gastos no bloco operatório, sem preocupações com a constituição da equipa cirúrgica, tempos alocados àquela cirurgia, entre outros, mas que são considerados no ABC.

A ligação entre o número do processo clínico de cada doente, onde constam os atos realizados e os consumos à base de dados onde constam os custos de cada acto/ consumo (custeio $A B C$ ) permite obter os custos reais 'mês*doente'.

O Hospital, ao longo do ano de 2014, recebeu a quantia mensal fixa de 929,08 € por doente, para o primeiro ano de financiamento do cancro de mama, independentemente do estádio $0^{6,8,9}$ da doença e consequentemente do consumo de recursos.

Os doentes foram agrupados de acordo com o respetivo estádio da doença, em linha com as normas de classificação TNM utilizadas na Instituição.

O modelo de tratamento, no IPO Lisboa, dos casos com cancro de mama tem uma abordagem clínica assente em duas fases. A fase I consiste na identificação dos fatores que enquadram e dão contexto ao modelo de tratamento. Estes fatores podem ser de ordem clínica e de ordem patológica. Os fatores de ordem clínica incluem o doente, a localização, o tamanho, a extensão local, a extensão regional e a extensão à distância. Os fatores de ordem patológica incluem os histológicos e os imuno-histoquímicos. A fase II consiste na escolha da opção terapêutica. Esta pode ter três tipologias: a) Tratamento cirúrgico - que tipo de cirurgia; b) Tratamento com radiações - qual, que dose, que modalidade e que área; c) Tratamento médico - quimioterapia: que esquema; hormonoterapia: qual; e imunoterapia: qual.

Tabela 1 - Caracterização da amostra (807 doentes) por idade e por sexo

\begin{tabular}{|c|c|c|c|c|c|c|c|}
\hline Grupo etário & Idade & $\%$ & Feminino & $\%$ & Masculino & $\%$ & Total geral \\
\hline$[25 ; 30]$ & 25 & $0,12 \%$ & 1 & $100 \%$ & 0 & $0, \%$ & 1 \\
\hline$[30 ; 35]$ & 30 & $1,86 \%$ & 15 & $100 \%$ & 0 & $0 \%$ & 15 \\
\hline$[35 ; 40]$ & 35 & $4,09 \%$ & 33 & $100 \%$ & 0 & $0 \%$ & 33 \\
\hline$[40 ; 45]$ & 40 & $8,05 \%$ & 65 & $100 \%$ & 0 & $0 \%$ & 65 \\
\hline$[45 ; 50]$ & 45 & $11,90 \%$ & 96 & $100 \%$ & 0 & $0 \%$ & 96 \\
\hline$[50 ; 55]$ & 50 & $11,28 \%$ & 91 & $100 \%$ & 0 & $0 \%$ & 91 \\
\hline$[55 ; 60]$ & 55 & $11,65 \%$ & 92 & $97,87 \%$ & 2 & $2,13 \%$ & 94 \\
\hline$[60 ; 65]$ & 60 & $9,91 \%$ & 80 & $100 \%$ & 0 & $0 \%$ & 80 \\
\hline$[65 ; 70]$ & 65 & $13,75 \%$ & 109 & $98,20 \%$ & 2 & $1,80 \%$ & 111 \\
\hline$[70 ; 75]$ & 70 & $9,05 \%$ & 73 & $100 \%$ & 0 & $0 \%$ & 73 \\
\hline$[75 ; 80]$ & 75 & $9,17 \%$ & 73 & $98,65 \%$ & 1 & $1,35 \%$ & 74 \\
\hline$[80 ; 85]$ & 80 & $6,07 \%$ & 46 & $93,88 \%$ & 3 & $6,12 \%$ & 49 \\
\hline $85+$ & 85 & $3,10 \%$ & 25 & $100 \%$ & 0 & $0 \%$ & 25 \\
\hline Total geral & & $100 \%$ & 799 & $99,01 \%$ & 8 & $0,99 \%$ & 807 \\
\hline
\end{tabular}


Tabela 2 - Custo total por estádio do cancro de mama ao longo dos meses financiados. Ano 2014.

\begin{tabular}{|c|c|c|c|c|c|c|c|}
\hline Estadiamento & Feminino & $\%$ & Masculino & $\%$ & Número de doentes & \multicolumn{2}{|c|}{ Custo total por estádio } \\
\hline 0 & 80 & $100 \%$ & 0 & $0 \%$ & 80 & \multicolumn{2}{|c|}{$308309,27 €$} \\
\hline I & 289 & $99,31 \%$ & 2 & $0,69 \%$ & 291 & \multicolumn{2}{|c|}{$1773516,72 €$} \\
\hline$\| A$ & 164 & $98,80 \%$ & 2 & $1,20 \%$ & 166 & \multicolumn{2}{|c|}{$1451963,33 €$} \\
\hline IIB & 114 & $100 \%$ & 0 & $0 \%$ & 114 & \multicolumn{2}{|c|}{$1287937,64 €$} \\
\hline IIIA & 55 & $98,21 \%$ & 1 & $1,79 \%$ & 56 & \multicolumn{2}{|c|}{$626485,23 €$} \\
\hline IIIB & 32 & $94,12 \%$ & 2 & $5,88 \%$ & 34 & \multicolumn{2}{|c|}{$365125,06 €$} \\
\hline IIIC & 19 & $100 \%$ & 0 & $0 \%$ & 19 & \multicolumn{2}{|c|}{$304755,91 €$} \\
\hline IV & 21 & $100 \%$ & 0 & $0 \%$ & 21 & \multicolumn{2}{|c|}{$305253,52 €$} \\
\hline Desconhecido & 25 & $96,15 \%$ & 1 & $3,85 \%$ & 26 & \multicolumn{2}{|c|}{$186470,28 €$} \\
\hline \multirow[t]{3}{*}{ Total } & 799 & $99,01 \%$ & 8 & $0,99 \%$ & 807 & \multicolumn{2}{|c|}{$6609816,96 €$} \\
\hline & & \multicolumn{3}{|c|}{$\begin{array}{c}\text { Custo total } \\
6609816,96 € \\
807 \text { Doentes }\end{array}$} & & \\
\hline & & $\downarrow$ & & & $\downarrow$ & $\downarrow$ & $\nabla$ \\
\hline \multicolumn{2}{|c|}{$\begin{array}{c}\text { Atendimento não programado } \\
\qquad \begin{array}{c}110084,43 € \\
1,7 \%\end{array}\end{array}$} & $\begin{array}{c}\text { Hospital de dia* } \\
3291168,20 € \\
49,8 \%\end{array}$ & \multicolumn{2}{|c|}{$\begin{array}{c}\text { Internamento } \\
567937,41 € \\
8,6 \%\end{array}$} & $\begin{array}{c}\text { Consultas externas } \\
\begin{array}{c}307585,51 € \\
4,7 \%\end{array}\end{array}$ & $\begin{array}{c}\text { MCDT's } \\
1721269,73 € \\
26,0 \%\end{array}$ & $\begin{array}{c}\text { Bloco } \\
611771,68 € \\
9,3 \%\end{array}$ \\
\hline
\end{tabular}

* Inclui ciclos de citotóxicos e imunomodeladores no valor de $2455284,51 €$

Fonte: própria dos autores

Os custos em Internamento totalizam o valor de 0,57 $\mathrm{M} €$, que representa $8,6 \%$ do valor global.

No Bloco Operatório, o total de custos foi de 0,6 M€, representando 9,3\% do valor global (Tabela 4).

No Atendimento Não Programado, os custos totalizam o valor de $0,1 \mathrm{M} €$, que corresponde a $1,7 \%$ da totalidade dos custos.

Constata-se que $41 \%$ da totalidade destes doentes realizaram tratamento em Hospital de Dia.

Concluiu-se, também, que nesta área de atendimento (Hospital de Dia) o maior número de doentes está nos estádios I, IIA e IIB (Tabela 5).

O número médio de sessões de hospital de dia por doente é mais elevado nos estádios IIIC e IV.

Nesta área, os custos são de 0,8 M€, aos quais acrescem os custos associados aos medicamentos utilizados nos ciclos de citotóxicos e imunomodeladores (2,5 M€) totalizando um custo de 3,3 M€, verificando-se assim que $50 \%$ dos custos globais são relativos ao hospital de dia, devido principalmente ao elevado custo dos medicamentos imunomodeladores.

O total de custos foi de 6,6 M€ e o total de financiamento foi de 5,2 M€, para um total de 5648 'meses*doente' (Tabela 6).

Tabela 3 - Custos e atividade com consultas externas, meios complementares de diagnóstico e terapêutica e internamento

\begin{tabular}{|c|c|c|c|c|c|c|}
\hline \multicolumn{7}{|c|}{ Custo total $=6609816,96 €$} \\
\hline \multicolumn{3}{|c|}{ Consultas externas $=307585,51 €(4,7 \%)$} & \multicolumn{2}{|c|}{ MCDT's $^{*}=1721269,73 €(26 \%)$} & \multicolumn{2}{|c|}{ Internamento $=567937,41 €(8,6 \%)$} \\
\hline Estadiamento & $\begin{array}{l}\text { Média de consultas } \\
\text { por doente }\end{array}$ & $\begin{array}{l}\text { Custo total consultas } \\
\qquad \mathrm{ABC}^{* *}\end{array}$ & $\begin{array}{l}\text { Número médio de } \\
\text { MCDT's* por doente }\end{array}$ & $\begin{array}{l}\text { Custo total MCDT's* } \\
\qquad \mathrm{ABC}^{* *}\end{array}$ & $\begin{array}{l}\text { Demora média } \\
\text { por doente }\end{array}$ & $\begin{array}{l}\text { Custo total } \\
\text { internamento } A B C^{* *}\end{array}$ \\
\hline 0/TIS & 15,3 & $15575,12 €$ & 60,1 & $171430,04 €$ & 2,3 & $45250,31 €$ \\
\hline 1 & 16,7 & $85397,98 €$ & 82,7 & $618088,75 €$ & 2,0 & $170200,06 €$ \\
\hline IIA & 19,4 & $66734,53 €$ & 120,5 & $294897,02 €$ & 3,7 & $129207,08 €$ \\
\hline IIB & 24,0 & $56803,81 €$ & 152,2 & $297179,70 €$ & 3,9 & $112681,08 €$ \\
\hline IIIA & 22,4 & $27365,18 €$ & 143,0 & $118410,62 €$ & 3,6 & $38110,07 €$ \\
\hline IIIB & 19,6 & $18450,98 €$ & 147,5 & $68951,65 €$ & 4,8 & $16372,97 €$ \\
\hline IIIC & 26,8 & $12948,37 €$ & 188,2 & $57529,24 €$ & 3,5 & $13075,21 €$ \\
\hline IV & 16,8 & $13887,26 €$ & 268,0 & $46735,64 €$ & 10,6 & $14900,10 €$ \\
\hline Desconhecido & 18,3 & $10422,28 €$ & 106,1 & $48047,07 €$ & 3,4 & $28140,54 €$ \\
\hline Total & 19,0 & $307585,51 €$ & 112,9 & $1721269,73 €$ & 3,1 & $567937,41 €$ \\
\hline
\end{tabular}

* Meios complementares de diagnóstico e terapêutica; ** Activity based costing 
Tabela 4 - Custos e atividade com bloco operatório

\begin{tabular}{|c|c|c|c|c|c|c|}
\hline \multicolumn{7}{|c|}{ Custo total $=6609816,96 €$} \\
\hline \multicolumn{7}{|c|}{ Bloco $=611771,68 €(9,3 \%)$} \\
\hline Estadiamento & $\begin{array}{l}\text { Número de } \\
\text { doentes }\end{array}$ & $\begin{array}{l}\text { Número de doentes } \\
\text { com bloco }\end{array}$ & $\begin{array}{l}\text { Número de } \\
\text { episódios }\end{array}$ & $\begin{array}{l}\text { Número de episódios } \\
\text { por doente }\end{array}$ & $\begin{array}{l}\text { Custo total bloco } \\
\qquad \mathrm{ABC}^{*}\end{array}$ & $\begin{array}{l}\text { Custo médio por } \\
\text { episódio }\end{array}$ \\
\hline 0/TIS & 80 & 61 & 75 & 1,2 & $61001,28 €$ & $813,35 €$ \\
\hline I & 291 & 209 & 203 & 1,1 & $220026,64 €$ & $1083,88 €$ \\
\hline$\| A$ & 166 & 122 & 155 & 1,3 & $135858,23 €$ & $876,50 €$ \\
\hline IIB & 114 & 83 & 118 & 1,4 & $102454,43 €$ & $868,26 €$ \\
\hline IIIA & 56 & 38 & 50 & 1,3 & $44542,92 €$ & $890,86 €$ \\
\hline IIIB & 34 & 15 & 19 & 1,3 & $15062,70 €$ & $792,77 €$ \\
\hline IIIC & 19 & 14 & 17 & 1,2 & $16.397,75 €$ & $964,57 €$ \\
\hline IV & 21 & 6 & 8 & 1,3 & $5059,45 €$ & $632,43 €$ \\
\hline Desconhecido & 26 & 13 & 14 & 1,1 & $11368,27 €$ & $812,02 €$ \\
\hline Total & 807 & 561 & 686 & 1,2 & $611771,68 €$ & $891,80 €$ \\
\hline \multicolumn{7}{|c|}{ Custo de bloco por doente $=1091 €$} \\
\hline \multicolumn{7}{|c|}{ Custo de bloco por Episódio $=892 €$} \\
\hline
\end{tabular}

${ }^{*}$ Activity based costing

Fonte: própria dos autores

Tabela 5 - Custos e actividade em hospital de dia

Custo total $=6609816,96 €$

Hospital de dia $=3291168,20 €(49,8 \%)$

\begin{tabular}{|c|c|c|c|c|c|c|}
\hline Estadiamento & $\begin{array}{c}\text { Número de } \\
\text { doentes com } \mathrm{HD}^{*}\end{array}$ & $\begin{array}{c}\text { Número de } \\
\text { sessões de } H D^{*}\end{array}$ & $\begin{array}{l}\text { Número médio de sessões } \\
\text { de HD* por doente }\end{array}$ & $\begin{array}{l}\text { Custo parcial } \\
H D^{*} A B C^{* *}\end{array}$ & $\begin{array}{c}\text { Ciclos de QT§ e } \\
\text { IM§§ }\end{array}$ & $\begin{array}{l}\text { Custo total HD* } \\
\left(\mathrm{ABC}^{* *}+\text { ciclos }\right)\end{array}$ \\
\hline 0/TIS & 0 & 0 & 0,0 & $0,00 €$ & $0,00 €$ & $0,00 €$ \\
\hline I & 70 & 342 & 4,9 & $161031,81 €$ & $471480,80 €$ & $632512,60 €$ \\
\hline IIA & 88 & 430 & 4,9 & $201844,45 €$ & $592797,50 €$ & $794641,95 €$ \\
\hline IIB & 68 & 397 & 5,8 & $187030,58 €$ & $547303,73 €$ & $734334,32 €$ \\
\hline IIIA & 39 & 209 & 5,4 & $98478,69 €$ & $288127,15 €$ & $386605,85 €$ \\
\hline IIIB & 26 & 132 & 5,1 & $60813,80 €$ & $181975,05 €$ & $242788,85 €$ \\
\hline IIIC & 16 & 111 & 6,9 & $52470,26 €$ & $153024,47 €$ & $205494,72 €$ \\
\hline IV & 14 & 116 & 8,3 & $53415,08 €$ & $159917,46 €$ & $213332,55 €$ \\
\hline Desconhecido & 9 & 44 & 4,9 & $20799,02 €$ & $60658,35 €$ & $81457,37 €$ \\
\hline Total & 330 & 1781 & 5,4 & $835883,69 €$ & $2455284,51 €$ & $3291168,20 €$ \\
\hline
\end{tabular}

* Hospital de dia; ** Activity based costing; § Quimioterapia; §§ Imunoterapia

Fonte: própria dos autores

\section{DISCUSSÃO}

Esta investigação tornou possível iniciar a monitorização dos outcomes em saúde, com a construção de séries cronológicas de dados sobre os tipos de tratamentos em cada estádio da doença, os custos associados e a construção de indicadores de resultados, como por exemplo: as taxas de sobrevivência por estádio da doença, os custos com medicamentos por estádio da doença, a demora média por episódio de internamento e por estádio da doença e a relação entre os custos e o financiamento.

No ano de 2014 foram financiados 5648 'meses*doente' na patologia do cancro de mama.

Através da análise realizada aos custos reais ocorridos pelos doentes incluídos no novo financiamento por patologia e utilizando o custeio-ABC, verificamos uma diferença substantiva entre custos reais e o montante financiado, existindo um desvio negativo de 1,4 $\mathrm{M} €$, isto é, em média cerca de $241,21 €$ por 'mês*doente' face ao 'envelope financeiro' fixo mensal de 929,08 €.

Nos estádios 0/TIS e I a soma dos custos associados ao tratamento dos doentes apresenta uma diferença positiva face ao financiamento obtido. ${ }^{9}$ Todos os outros estádios geraram uma diferença negativa.

As situações extremas em termos de custos por 'mês*doente' face ao 'envelope financeiro' mensal fixo foram o estádio 0/TIS com financiamento superior em 415,23 € por 'mês*doente' e o estádio IIIC com um financiamento inferior em 1062,79 € por 'mês*doente'.

As diferenças identificadas nesta investigação, entre o que são os custos reais do cancro de mama e o montante financiado, demonstram um desajustamento do financiamento em relação aos custos reais no standard de 
Tabela 6 - Doentes entrados ao longo do ano 2014, respetivos meses de financiamento e custos reais incorridos 'mês * doente'

\begin{tabular}{|c|c|c|c|c|c|c|c|}
\hline Estádio & $\begin{array}{c}\mathrm{N}^{\circ} \text { de } \\
\text { doentes }\end{array}$ & $\begin{array}{l}\text { Custo total } \\
\text { ABC }\end{array}$ & Financiamento & $\begin{array}{c}\Delta \text { financiamento/ } \\
\text { custos }\end{array}$ & $\begin{array}{l}\text { 'Meses } \\
\text { doente' }\end{array}$ & $\begin{array}{c}\text { Custo } \\
\text { 'mês doente' }\end{array}$ & $\begin{array}{l}\Delta \text { financiamento/ } \\
\text { custos 'mês doente' }\end{array}$ \\
\hline 0 & 80 & $308309,27 €$ & $557448,00 €$ & $249138,73 €$ & 600 & $513,85 €$ & $415,23 €$ \\
\hline I & 291 & $1773516,72 €$ & $1856301,84 €$ & $82785,12 €$ & 1998 & $887,65 €$ & $41,43 €$ \\
\hline IIA & 166 & $1451963,33 €$ & $1051718,56 €$ & $-400244,77 €$ & 1132 & $1282,65 €$ & $-353,57 €$ \\
\hline IIB & 114 & $1287937,64 €$ & $770207,32 €$ & $-517730,32 €$ & 829 & $1553,60 €$ & $-624,52 €$ \\
\hline IIIA & 56 & $626485,23 €$ & $353050,40 €$ & $-273434,83 €$ & 380 & $1648,65 €$ & $-719,57 €$ \\
\hline IIIB & 34 & $365125,06 €$ & $211830,24 €$ & $-153294,82 €$ & 228 & $1601,43 €$ & $-672,35 €$ \\
\hline IIIC & 19 & $304755,91 €$ & $142149,24 €$ & $-162606,67 €$ & 153 & $1991,87 €$ & $-1062,79 €$ \\
\hline IV & 21 & $305253,52 €$ & $174667,04 €$ & $-130586,48 €$ & 188 & $1623,69 €$ & $-694.61 €$ \\
\hline Desconhecido & 26 & $186470,28 €$ & $130071,20 €$ & $-56399,08 €$ & 140 & $1331,93 €$ & $-402,85 €$ \\
\hline Total & 807 & $6609816,96 €$ & $5247443,84 €$ & $-1362373,12 €$ & 5648 & $1170,29 €$ & $-241,21 €$ \\
\hline
\end{tabular}

Fonte: própria dos autores

tratamento desta doença no IPO Lisboa, tratamento que segue a atual legis arte, estando em linha com outros estudos sobre sub-finaciamento do tratamento do cancro em Portugal. ${ }^{10}$

Um vetor que importa ressalvar nesta investigação, está relacionado com os medicamentos citotóxicos e imunomodeladores utilizados nas sessões de hospital de dia num total de 2,5 M€. Os medicamentos representam 38\% dos custos globais envolvidos (6,6 M€), em 2014, e que muito provavelmente não estão contemplados na formulação deste financiamento por patologia. Este rácio está em linha com o assinalado em outros estudos relacionados com a despesa de medicamentos no tratamento do cancro. ${ }^{11}$

O estudo em apreço tem algumas limitações, desde logo por não ser conhecida a formulação do pricing $929,08 €$ fixo mensal por doente. Depois por não terem sido encontrados outros estudos que permitissem comparação, apenas alguma aproximação de estimativas internacionais. Ou seja, os estudos sobre a patologia da mama não se encontram estruturados nem metodologicamente, nem em termos conceptuais de forma a permitir uma analogia ao presente estudo.

Também não foram encontrados estudos que desagreguem os custos por estádios TNM. Destarte, foi contudo identificado um estudo realizado no Reino Unido, ${ }^{7}$ ao longo de 15 meses (anos 2010 e 2011) com uma amostra de 223 pacientes com cancro da mama e os custos por ano e por doente foram de $11870,83 €$.

Este valor permite obter um custo 'mês*doente' de 989,24 $€$ desconhecendo-se, no entanto os estádios da doença destes 223 doentes. Todavia se compararmos com o valor que resulta do presente estudo de $1170,29 €$ chegamos a uma diferença de $181,05 €$ superior ao do Reino Unido $^{7}$.

\section{CONCLUSÃO}

Este estudo pretendeu chamar a atenção para a necessidade do conhecimento dos custos reais por doente, por patologia e por estádio da doença.

No caso estudado, o cancro de mama, os custos por estádio da doença podem vir a ser mais relevantes para a formulação do pricing em saúde, do que apenas os custos por patologia sem qualquer detalhe por estádio.

A metodologia $A B C$ utilizada nesta investigação vem proporcionar uma nova visão e, desde já, um envolvimento profissional e multidisciplinar único, conseguido pela aproximação da linguagem clínica à linguagem de gestão.Esta linguagem comum é um elemento facilitador do processo de decisão e da partilha de dados, de informação e de conhecimento, esbatendo o status quo que durante décadas esteve instalado entre as práticas clínicas e as práticas organizativas que se moviam entre 'muros', por desconhecimento dos termos e dos conceitos utilizados em cada uma destas áreas científicas.

As dimensões abordadas foram: (i) a elegibilidade dos doentes de acordo com os critérios do novo programa de financiamento por patologia iniciado no ano de 2014; (ii) os custos reais 'mês*doente' e por estádio da doença tendo em conta os critérios clínicos de tratamento (iii) o 'envelope financeiro' fixo mensal para o cancro de mama.

O estudo, integrando estas dimensões, permitiu uma apreciação presente sobre a organização de cuidados e sobre a necessidade do desenvolvimento de novos estudos com a metodologia de custeio $A B C$, nomeadamente para estudos sobre eficiência dos serviços,,$^{12}$ sobre a qualidade das prestações, ${ }^{13}$ sobre a adequação do financiamento, mas também para futura monitorização dos outcomes em saúde.

Esta investigação permitiu, ainda, refletir sobre a população que o IPO Lisboa trata, constatando-se que a mesma é representada por todo o espectro de estádios, acompanhando as séries internacionais, e não apenas por doentes em estádios mais avançados, consequentemente mais graves, que poderiam implicar custos totais mais elevados que os apurados.

O aumento dos custos em oncologia deve-se ao aumento da incidência do cancro ao longo dos anos ${ }^{14,15} \mathrm{e}$ às novas indicações terapêuticas com fármacos já disponíveis no mercado que são mais dispendiosos.

O modelo de financiamento, ${ }^{16}$ para ser realista, deve acomodar além dos estádios da doença, as práticas inovadoras de tratamento. A título de exemplo e para o caso em 
estudo, realiza-se, num único tempo operatório, a cirurgia de recessão de mama imediatamente seguida da cirurgia reconstrutiva, melhorando assim, a auto-imagem e a qualidade de vida dos doentes. Todavia, estas inovações devem ser abordadas nos modelos de financiamento como substitutivas ou alternativas às anteriores práticas e o respetivo impacto económico das mesmas.

Para concluir há necessidade de estudar e aprofundar a medição de custos através do custeio $A B C$, essencial para estabelecer modelos de financiamento adequados e orientados para os outcomes, ${ }^{17}$ crucial para a sustentabilidade futura do sector da saúde. ${ }^{18}$

\section{PROTECÇÃO DE PESSOAS E ANIMAIS}

Os autores declaram que os procedimentos seguidos estavam de acordo com os regulamentos estabelecidos

\section{REFERÊNCIAS}

1. Organização Mundial de Saúde. Fact Sheet $n^{\circ}$ 297. Genebra: OMS; 2015.

2. Dados do Registo Oncológico Nacional, relativo ao ano de 2010 . Elaborado pelo Registo Oncológico Regional do Norte. Porto: Instituto Português de Oncologia do Porto Francisco Gentil - EPE; 2016.

3. Costa S, Jimenéz F, Ribeiro J. Imagem corporal, sexualidade e qualidade de vida no cancro da mama. Psicol Saúde Doenças.2012;13: 2.

4. Borges C, Ramalho R, Bajanca M, Oliveira T, Major M, Diz P, et al. Implementação de um sistema de custeio por actividades nos hospitais do SNS. Rev Port Saúde Pública. 2010;9:141-55.

5. Kaplan RS, Cooper R. Cost \& effect. Boston: Harvard Business School Press; 1998.

6. Greene F, Page D, Fleming I, Fritz A, Balch C, Haller D, et al. American Joint Committee on Cancer Staging Manual, Cancer Staging Handbook. $7^{\mathrm{a}}$ ed. Chicago: Springer; 2010

7. Hall $P$, Hamilton $P$, Hulme $C$, Meads $D$, Jones $H$, Newsham A, et al. Costs of cancer care for use in economic evaluation: a UK analysis of patient-level routine health system data. Br J Cancer. 2015;112:948-56.

8. BREASTCANCER.ORG. [consultado 2015 jun 22]. Disponível em: http://www.breastcancer.org/symptoms/diagnosis/staging consultado a 22/06/2015.

9. Administração Central do Sistema de Saúde. Contracto Programa de 2013. Lisboa: ACSS; 2012 pelos responsáveis da Comissão de Investigação Clínica e Ética e de acordo com a Declaração de Helsínquia da Associação Médica Mundial.

\section{CONFIDENCIALIDADE DOS DADOS}

Os autores declaram ter seguido os protocolos do seu centro de trabalho acerca da publicação de dados.

\section{CONFLITOS DE INTERESSE}

Os autores declaram não terem qualquer conflito de interesse relativamente ao presente artigo.

\section{FONTES DE FINANCIAMENTO}

Os autores declaram não ter recebido subsídios ou bolsas para a elaboração do artigo.

10. Araújo A, Barata F, Barroso $S$, Cortes $P$, Damasceno $M$, Parreira A, et al. Custo do tratamento do cancro em Portugal. Acta Med Port. 2009;22:525-36.

11. Uramoto H, Kagami S, Iwashige A, Tsukada J. Evaluation of the quality of life between inpatients and outpatients receiving cancer chemotherapy in Japan. Anticancer Res. 2007;27:1127-32.

12. Harfouche A. Hospitais transformados em empresas - análise do impacto na eficiência: estudo comparativo. Lisboa: ISCSP; 2008.

13. The Organisation for Economic Co-operation and Development. OECD Reviews of Health Care Quality: Portugal 2015: Raising Standards. Paris: OECD; 2015.

14. World Health Organisation. Health Statistics 2015. Geneve: WHO; 2015.

15. Ferlay J, Steliarova-Foucher E, Lortet-Tieulent J, Rosso S, Coeburgh JW, et al. Cancer incidence and mortality patterns in Europe: estimates for 40 countries in 2012. Eur J Cancer. 2013;1374-1403.

16. Harfouche A. Políticas de saúde: fundamentação, prioridades, opções e resultados. Lisboa: ISCSP; 2016.

17. Harfouche A. Opções políticas em saúde: efeitos sobre a eficiência hospitalar. Coimbra: Almedina; 2012.

18. The Organisation for Economic Co-operation and Development. Tackling wasteful spending on health - learning from OECD countries' experience. Paris: OECD; 2017. 